\title{
Preparation and Characterization of Polydimethylsiloxane- based Paper Transparentizing Agent and its Application in Paper Coating
}

\author{
Haoran Ma, Yujie Gao, * and Longgang Tian
}

\begin{abstract}
A new type of polydimethylsiloxane-based paper transparentizing agent was prepared via a combined method. The performance of the transparentizing agent was investigated systematically by adding and dipping, with use of the surface sizing device of a paper machine. Optimum performance was found at $30 \%$ concentration of the transparentizing agent and 30 to $45^{\circ} \mathrm{C}$ of dipping temperature. Under the optimal conditions, the transparentizing $t$ agent achieved a rapid penetration in the base paper and filled in the pores of the paper, to be further effectively adsorbed and retained on the fiber surface. The transparency of test paper reached as high as $76 \pm 0.97 \%$, which was $37 \pm$ $1.4 \%$ higher than that of the control. However, the addition of transparentizing agent reduced the mechanical strength of paper slightly. The as-prepared transparentizing agent was found to exhibit excellent application stability and biodegradability when applied in the paper machine, When used as plastic film, the transparent paper would lose its strength completely after eight weeks. The resulting transparent paper can be used to develop paper-based film and other related plant based transparent/ translucent paper, which has great potential in replacing plastic products and eliminating white pollution.
\end{abstract}

Keywords: Transparentizing agent; Transparency; Transparent paper; Film; Biodegradation

Contact information: Tianjin University of Science and Technology, Tianjin, China;

*Corresponding author: yujie@tust.edu.cn

\section{INTRODUCTION}

Potential applications of transparent paper products can be found in many fields. For instance, the use of transparent paper instead of traditional plastic films can be considered (Zhu et al. 2014). Mulch cultivation technology, such as agricultural mulch film, has been used in various areas of agricultural production (Cirujeda et al. 2013). It plays an essential role in maintaining soil temperature and moisture content as well as supporting plant roots (Marí et al. 2019). Mulch cultivation can maintain soil moisture, improve mineralization, and save water consumption (Sun et al. 2019; Ibrahim et al. 2020). However, large amounts of plastic film have been utilized with mulch cultivation technology, and such practices would generate lots of plastic waste and can cause serious white pollution due to the non-degradable properties of plastics (Shen et al. 2011). Furthermore, if these used mulch films are buried directly into the soil without additional treatment or purification process, the nature of the soil would be changed and the root system of the next batch of crops would also be affected, thus resulting in infertility soil and environmental pollution (Cirujeda et al. 2012; Li et al. 2018). Transparent paper-based mulch film derived from natural plant fibers can be used as a kind of new type of paperbased products that can be environmentally friendly and easily degradable (Minoru and Yamauch 2001). 
Light can significantly influence the seed germination and seedling growth, thereby ensuring that young plants are exposed to an optimal environment for photosynthesis. (Jang et al. 2008). However, the traditional paper-based products have a low transparency due to their high bulk property. The most common method to produce transparent or translucent paper is to increase the beating degree of cellulose fibers prepared for papers, and to thereby increase the density and reduce the internal pores of paper product with a low bulk (Mackey et al. 1994). However, this method requires strict selection of pulp raw materials, and it needs additional beating time and energy consumption (Chen et al. 2012). Pulp with a high beating degree is difficult to be dewatered during the paper-making process of transparent paper production, thus greatly limiting the production and application of transparent paper (Zhou et al. 2018a). Recently, nano-cellulose has been applied as a raw material for the preparation of nanopaper with high transparency (Su et al. 2018). However, because of the nano size and its strong retention ability of water, nano-cellulose requires a long time to dewater during the paper-making process, thus consuming more energy and causing high costs, which is not practical in the real production (Hassan et al. 2016).

Impregnating paper with a transparentizing agent to improve paper transparency is an effective method. Transparentizing agents have a similar refractive index to cellulose. When filled into the paper structure, transparentizing agents can help unify the overall refractive index of the paper, and by that means increase the paper transparency (Chen et al. 2012). Most commonly used transparentizing agents are resins, oils, synthetic substances, and paraffin wax (Li et al. 2019). The traditional preparation method that relies on a transparentizing agent from only one raw material cannot meet the strict industrial demands (Vitz et al. 2010). In this study, different materials (modified polydimethylsiloxane, varnish and paraffin, etc.) would be utilized with various properties to prepare a novel transparentizing agent emulsion with high efficiency (Abbasi et al. 2001). Transparent paper agent was applied by combining with conventional papermaking methods to produce degradable transparent paper with low production cost and high transparency (Tiwari and Mahanwar 2018). The transparency of test paper reached as high as $76 \pm 0.97 \%$, which was $37 \pm 1.4 \%$ higher than that of the control. The transparent paper meets the strict requirements during the utilization as paper base films, instead of traditional plastic film, and can effectively solve the severe white pollution issue. The transparent paper's base weight lost $52.92 \pm 7.35 \%$ after 6 weeks' treatment in an outdoor environment. The development and production of agricultural mulch paper will have important theoretical and practical significance.

\section{EXPERIMENTAL}

\section{Materials}

The main agents were modified polydimethylsiloxane (BYK-310, Kingchemical Co., Ltd, Shanghai, China), varnish (xsxd-002 Guangdong Shunde Bejuly New Material Co., Ltd, Guangdong, China), and paraffin (P104801, Tianjin Jiangtian Chemical Technology Co., Ltd, Tianjin, China).

Bleached softwood pulp was provided by the specialty paper research group in Tianjin University of Science and Technology, China. Deionized (DI) water was utilized in the experiment.

All other chemicals such as emulsifying agents (9041-29-6 Sanjiang sairuida Trading Co., Ltd, Tianjin, China), curing agents (MD-50, Laiqing new materials Co., Ltd, 
Tianjin, China), PAE (MD-50, Laiqing new materials Co., Ltd, Tianjin, China) and penetrating agents were of analytical grade and used without further purification (Pelton $e t$ al. 2019).

\section{Methods}

Preparation of transparentizing agent

Modified polydimethylsiloxane was applied to prepare the transparentizing agent by an integrated method. The paraffin mixture and varnish was heated to $60{ }^{\circ} \mathrm{C}$ in a water bath (in a 3:2 ratio), followed by the addition of emulsifier and modified polydimethylsiloxane into the above mixture (Tian et al. 2015). Deionized water was added slowly (pre-heated to $60{ }^{\circ} \mathrm{C}$ ) under magnetic stirring. The mixture was emulsified for 10 min to ensure a stable emulsion (Taiki and Masami 2012). Afterwards, the curing agent and penetrating agent were subsequently added into the above mixture. At the end of reaction, the transparentizing agent was obtained by removing the heat and allowing the agent to cool down to room temperature (Guan et al. 2020).

\section{Preparation of transparent paper}

Bleach softwood pulp was beaten to $20{ }^{\circ} \mathrm{SR}$. A base paper sheet with $40 \mathrm{~g} / \mathrm{m}^{2}$ dry basis weight was prepared with a sheet former. During the papermaking process, $1 \%$ PAE wet strength agent was added on one side of the paper. Drying temperature was set to 80 ${ }^{\circ} \mathrm{C}$ with a drying time of $20 \mathrm{~min}$. The dipping method was used to prepare the transparent paper. The base paper was immersed in the transparentizing agent for a certain period (another group was impregnated with deionized water as control sample), the paper was lifted with a nylon drag, followed by removing the excess transparentizing agent from the paper sheet. The dipped paper sheet was dried with a drum dryer, and the dry transparent paper was obtained for further use (Syed et al. 2016).

\section{Analysis of transparentizing agent}

The viscosity of the transparentizing agent was determined by placing the transparentizing agent in the viscometer at $25{ }^{\circ} \mathrm{C}$, using the No. 1 rotor, adjusting the rotation speed to $120 \mathrm{rpm} / \mathrm{min}$, and measuring the emulsion viscosity until 5 stable readings were taken (Each of the measurement in this paper was calculated from the average of three parallel runs).

The refractive index was determined after drying and curing of the transparentizing agent in the paper sheet. After the transparentizing agent was dried on a flat surface to form a film, it was cut into an appropriate size and measured with an Abbe refractometer (Xuancheng Instrument Co., Ltd., Shanghai, China) (Koroleva et al 2017).

\section{Determination of paper transparency}

The paper transparency can be measured by the 070E Brightness Meter (L\&W Co., Ltd., Sweden). The average value of green light reflection factor of single layer specimen with white standard plate backing was designated as $R_{\mathrm{w}}$, and the average value of green light reflection factor of single layer specimen with black standard cylinder backing was designated as $R_{\mathrm{o}}$. The final transparency was calculated from the average of three parallel runs of measurements for error analysis. The transparency was given as follows:

$$
\text { transparency }(\%)=\frac{R_{w}-R_{o}}{R_{w}} \times 100 \%
$$




\section{Measurement of tensile strength of paper}

The tensile strength of paper sample was measured according to TAPPI test method T456 om-87 (L\&W Co., Ltd., Sweden). The tensile strength was calculated from the average of three parallel runs of measurements for error analysis.

\section{Measurement of bursting strength and tear index}

The bursting strength of paper was tested on a 13-60-00-0002 tester of bursting strength (TMI Co., Ltd., Sweden).

The tearing strength of paper was tested on a 009 tester of Paper and board-tearing tester (L\&W Co., Ltd., Sweden), and the width of paper sample was $1.5 \mathrm{~cm}$.

The final results were calculated from the average of three parallel runs of measurements for error analysis.

\section{Analyses of scanning electron microscope (SEM)}

For the SEM observation of transparent paper, pre-weighed samples were put on a desiccator overnight at room temperature. The samples then were mounted on a conductive carbon tape and coated with gold before the scanning process. The SEM pictures were captured using Oxford instrument with a $10 \mathrm{kV}$ beam (Seta et al. 2020).

\section{Biodegradability analyses of transparentizing agent}

Soil burial tests were performed on a laboratory scale to examine the biodegradability of the transparent paper-based film. The soil burial test method described by Obasi et al. (2013) was used with some modification. The crushed and sieved soil blocks were taken outdoors and placed in $2000 \mathrm{~mL}$ beakers. The transparent paper was cut into discs with a diameter of $160 \mathrm{~mm}$ and buried at a depth of $3 \mathrm{~cm}$ from the soil surface to ensure that the samples were completely buried in the soil. The soil was kept moist and watered every three days (Al-Kayssi et al. 2013). The soil burial test lasted for 90 days. After the test, the samples were removed, washed with distilled water, dried in the oven at $70{ }^{\circ} \mathrm{C}$ for $24 \mathrm{~h}$, then cooled to $20^{\circ} \mathrm{C}$ in a desiccator.

\section{RESULTS AND DISCUSSION}

\section{Effects of Transparentizing Agent Concentration on Its Application Effect} Effects of concentration of transparentizing agent on viscosity and transparency

The high viscosity of transparentizing agent improves its penetration speed in the base paper, which is of great significance for producing transparent paper at higher paper machine speeds (Chen et al. 2015). The viscosity and transparency of the prepared transparency at different concentrations are shown in Fig. 1. The immersion temperature was $20^{\circ} \mathrm{C}$, and the immersion time was $5 \mathrm{~s}$.

Figure 1 shows that the increased concentration of transparentizing agent increased the viscosity to a high level. When the concentration was in the range of $10 \%$ to $30 \%$, the viscosity increased slowly. At this time, the transparency of the finished paper was positively correlated with the viscosity, and it was assumed that the concentration of transparentizing agents has equal positive effect on the viscosity. When the concentration of transparentizing agent was more than $30 \%$, the viscosity increased greatly, but the transparency showed a downward trend. When the concentration of the transparentizing agent was in a lower range ( 0 to $30 \%$ ), the effects on the viscosity were not apparent. At 
this stage, the transparentizing agent always exhibited a low viscosity level. Thus, the penetration speed in the base paper was not affected significantly when the concentration of transparentizing agent did not exceed $30 \%$, i.e., the optimum concentration was $30 \%$ (Hämäläinen et al. 2011).

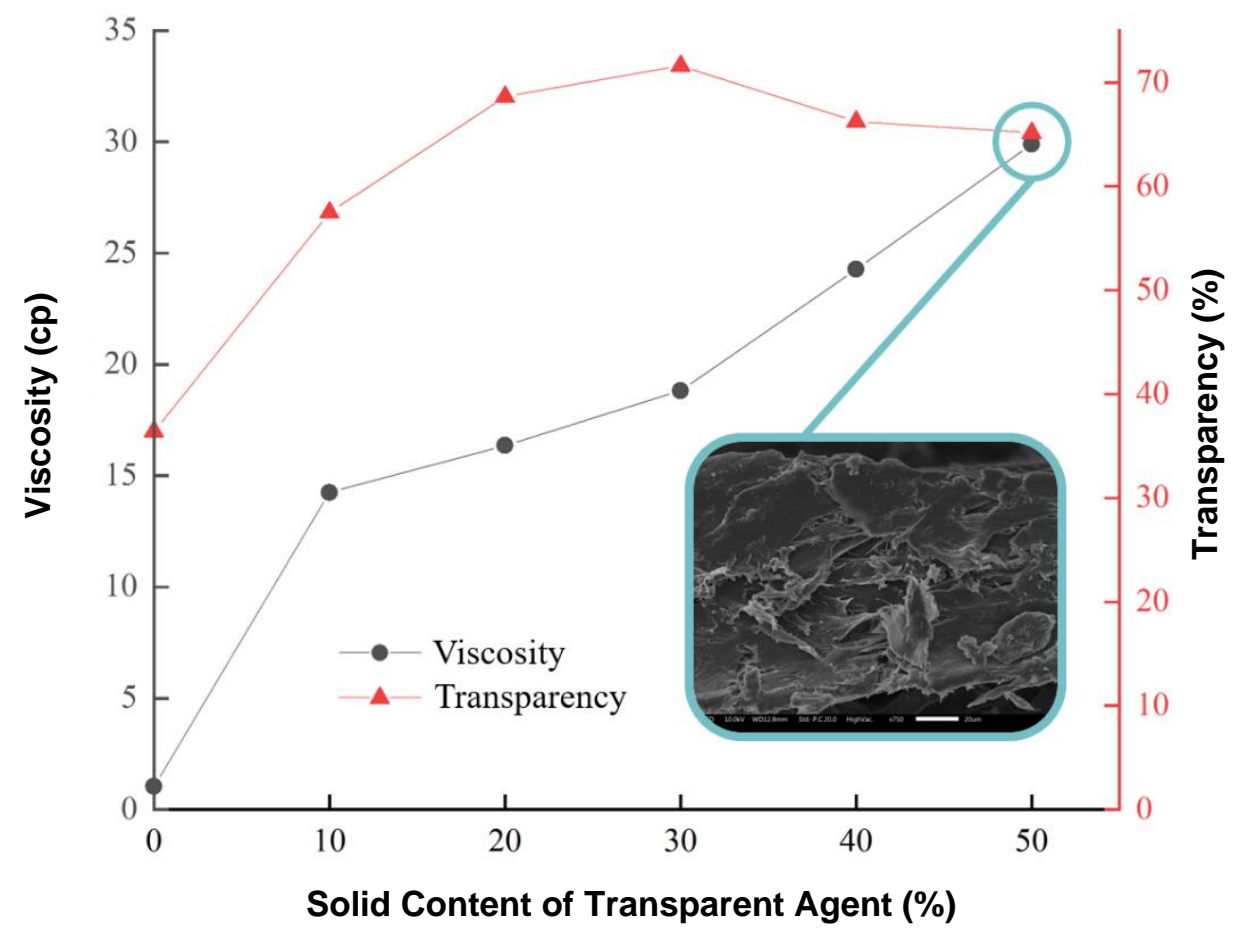

Fig. 1. Effects of concentration of transparentizing agent on the viscosity

Under the same immersion conditions, if the concentration of the transparentizing agent exceeded $30 \%$, the transparency began to decrease. At this time, the higher viscosity would limit the dipping speed of the transparentizing agent. SEM image shows that the fluidity and permeability of the transparentizing agent were insufficient to permeate into paper and bridge effects even appeared on the surface of the paper when at a $50 \%$ transparentizing agent concentration. The transparentizing agent was stacked on the surface of paper sheet and could not penetrate into the paper sheet. Figure 1 also shows that the transparency of the paper also decreased. It should be noted that when the concentration was higher than $20 \%$, the increasing trend of the paper transparency began to slow down. In the real production, the prepared transparentizing agent can precisely increase the transparency of paper sheets, thanks to the improved permeation ability of transparentizing agent into the paper sheet structure and fiber gaps (Zhenzhen et al. 2019).

\section{Effects of transparentizing agent concentration on the paper performance}

The base paper was dipped into transparentizing agent solutions with different concentrations under the same conditions $\left(40^{\circ} \mathrm{C}\right.$, immersion for $5 \mathrm{~s}$, and the same liquid level) to investigate the effects of transparentizing agents of different concentrations on the performance of the paper. The experimental results are shown in Table 1. 
Table 1. Effects of Transparentizing Agent Concentration on Paper Sheet Performance

\begin{tabular}{|c|c|c|c|c|c|c|}
\hline $\begin{array}{c}\text { Transparentizing } \\
\text { Agent } \\
\text { Concentration } \\
(\%)\end{array}$ & 0 & 10 & 20 & 30 & 40 & 50 \\
\hline $\begin{array}{c}\text { Basis weight } \\
\left(\mathrm{g} / \mathrm{m}^{2}\right)\end{array}$ & $41.60 \pm 2.75$ & $51.30 \pm 2.13$ & $58.40 \pm 4.06$ & $60.10 \pm 3.37$ & $52.00 \pm 3.06$ & $49.70 \pm 6.66$ \\
\hline Density $\left(\mathrm{g} / \mathrm{cm}^{3}\right)$ & $0.53 \pm 0.014$ & $0.69 \pm 0.007$ & $0.74 \pm 0.009$ & $0.75 \pm 0.006$ & $0.71 \pm 0.011$ & $0.67 \pm 0.003$ \\
\hline $\begin{array}{c}\text { Burst index } \\
\left(\mathrm{kPa} \cdot \mathrm{m}^{2} / \mathrm{g}\right)\end{array}$ & $4.37 \pm 0.24$ & $3.10 \pm 0.23$ & $3.03 \pm 0.19$ & $2.57 \pm 0.07$ & $2.60 \pm 0.21$ & $2.52 \pm 0.17$ \\
\hline $\begin{array}{c}\text { Tensile Index } \\
(\mathrm{N} \cdot \mathrm{m} / \mathrm{g})\end{array}$ & $52.83 \pm 1.36$ & $37.20 \pm 1.14$ & $32.41 \pm 0.85$ & $30.53 \pm 1.58$ & $30.12 \pm 0.61$ & $29.87 \pm 0.57$ \\
\hline $\begin{array}{c}\text { Tear index } \\
\left(\mathrm{mN} \cdot \mathrm{m}^{2} / \mathrm{g}\right)\end{array}$ & $8.31 \pm 0.26$ & $5.78 \pm 0.57$ & $4.81 \pm 0.19$ & $4.67 \pm 0.25$ & $5.37 \pm 0.51$ & $5.91 \pm 0.27$ \\
\hline
\end{tabular}

The transparency of paper sheet increased with the augment in the dipping amount, but simultaneously, the strength performance of the paper sheet including burst tensile and tear indexes decreased, which shows that the strength loss occurred when the transparent material filled the fiber gaps (Table 1). Although it increased the uniform refractive index in the paper sheet, the hydrogen bonding and the bridging effects between fibers would be blocked and weakened. Therefore, a suitable transparentizing agent concentration should be determined to ensure that at this concentration, the active substances in the transparentizing agent can fill into the inter-fiber pores and maintain the strength properties of the paper sheet. It can be concluded that the optimum transparentizing agent concentration was $30 \%$.

\section{Effects of Dipping Time on the Transparency Property}

Treatment of base paper to improve its transparency can be affected by various factors. The immersion time of paper sheet during the dipping process determines the retention rate of transparentizing agent in the voids of the paper sheet. It is understandable that extending the immersion time can facilitate a greater amount of transparentizing agent filling into the paper sheet. However, when the immersion time reaches a critical level, the transparentizing agent emulsion will fill into the internal pores of the paper. If the immersion time is overextended, the transparency of paper sheet won't be improved, but there can be a serious loss of paper strength, and the speed of the paper machine will be significantly reduced (Fang et al. 2013). Therefore, the effect of impregnation time on paper was explored. The base paper was prepared by transparentizing agent impregnation at $20{ }^{\circ} \mathrm{C}$ with $30 \%$ concentration of transparentizing agent dosage.

\section{Effects of dipping time on paper transparency}

Figure 2 showed that the transparency of the paper sheet increased rapidly in a short period during the initial stage of dipping (the dipping temperature was $40^{\circ} \mathrm{C}$ ), reaching $70.6 \pm 0.41 \%$ at $5 \mathrm{~s}$, which is higher compared to initial paper before dipping $(34.2 \pm 0.67 \%)$. After $5 \mathrm{~s}$, the pores in the paper were nearly saturated, the transparency of the paper sheet was unchanged and the transparency after immersion for $60 \mathrm{~s}$ was only increased by $2.6 \pm 0.83 \%$ with a dipping time of $5 \mathrm{~s}$. The transparentizing agent developed in this paper fully penetrated the paper sheet quickly, thus improving the transparency, which is beneficial to the practical application on the paper machine. 


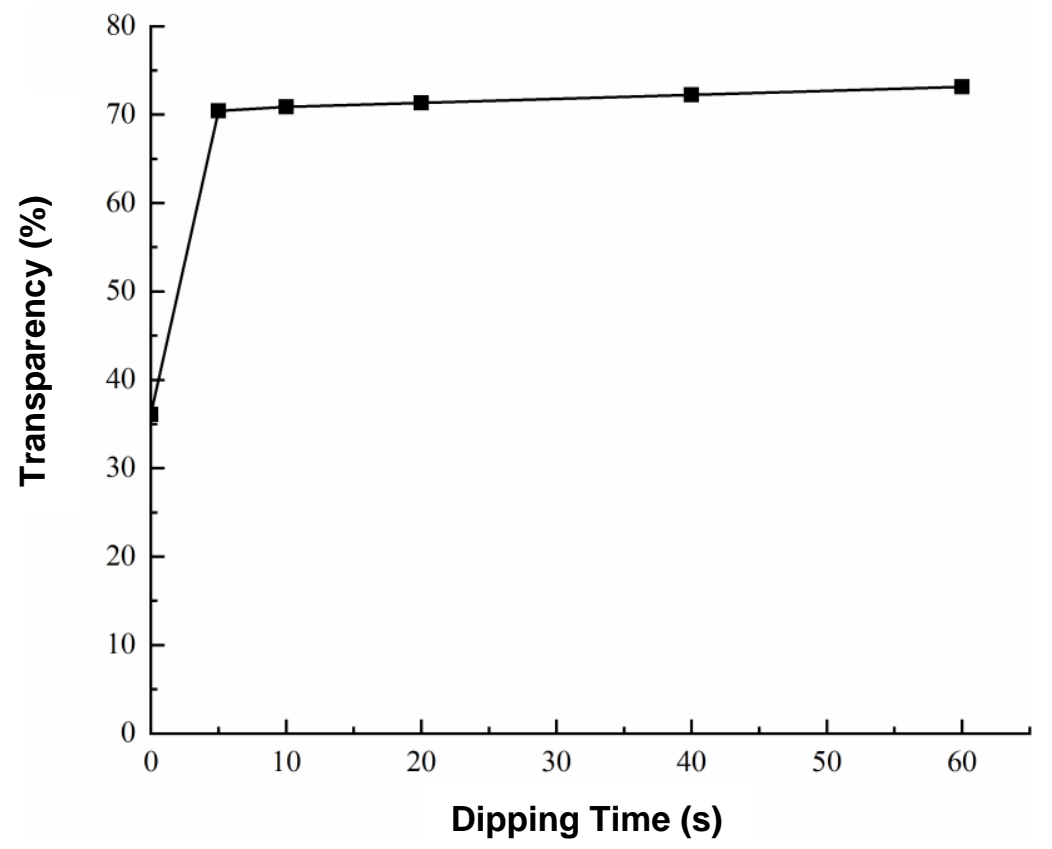

Fig. 2. Effects of dipping time on transparency of paper

Effects of dipping time on paper strength property

As shown in Fig. 3, with the increase of the immersion time in the agent, the strength properties, for example, tensile index of the paper sheet, were found to be decreased significantly, which can be ascribed to the breaking up/blocking effect of hydrogen bonding between fibers induced by the addition of transparentizing agent (Zhang et al. 2014). Besides, it was also found that the long-term immersion time can improve the moisture content of paper sheet, thus impeding the drying efficiency in the paper machine, which is undesirable in paper production (Zhou et al. 2018b). The optimal dipping time was $5 \mathrm{~s}$, considering the results shown in Fig. 3.

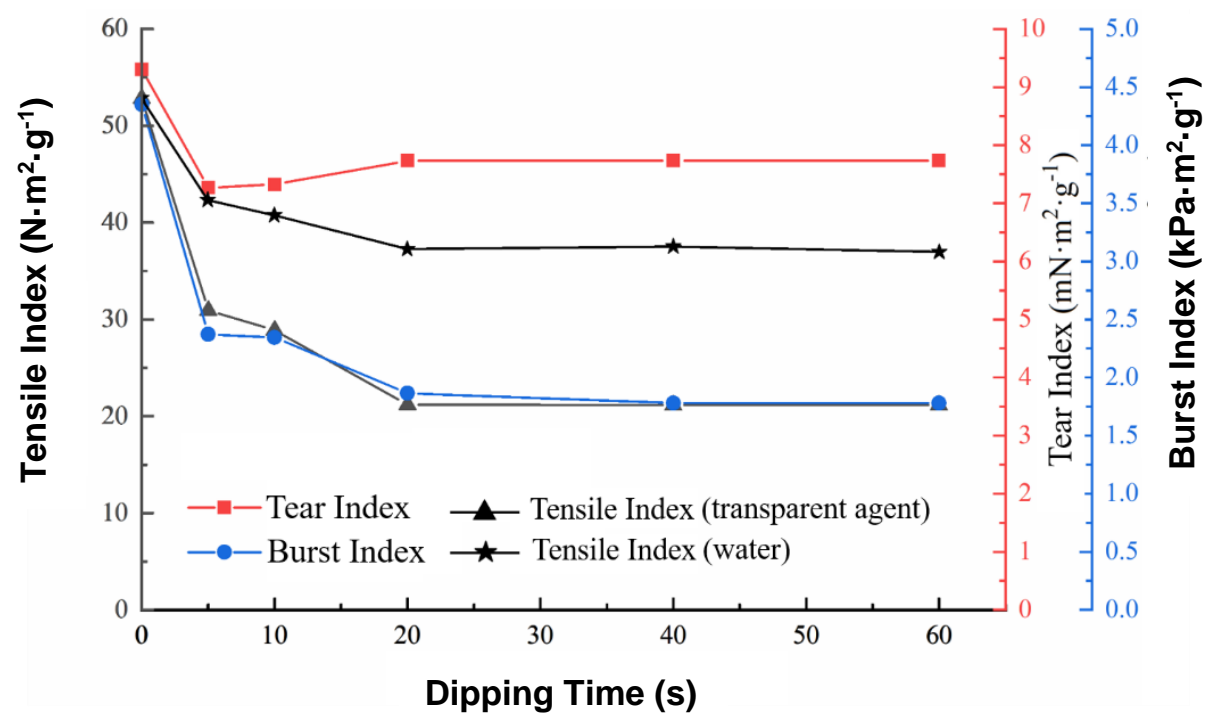

Fig. 3. Effects of dipping time on strength of paper 


\section{Effects of Dipping Temperature on the Transparency Performance of Paper Sheet}

Effect of dipping temperature on paper transparency

Experimental results in Fig. 4 showed that the transparency of paper sheet did not change significantly with the increasing of the dipping temperature in a specific range (the dipping time was $5 \mathrm{~s}$ ). The transparency of the paper sheet reached $76.04 \pm 1.44 \%$, which was $37.38 \pm 0.56 \%$ higher than that of the base paper. When the temperature exceeded $45^{\circ} \mathrm{C}$, the transparency decreased. Theoretically, higher coating temperatures could reduce the viscosity of the transparentizing agent, but results indicate the opposite. Because the emulsion contains varnish, and it will form a thin film on the liquid surface at a high temperature, then the refractive index of agent was changed. Alternatively, increasing temperature will accelerate the transparentizing agent's liquid phase components' volatilization, causing the transparentizing agent's viscosity to increase rapidly, which would dramatically affect the penetration efficiency within a limited time (Tang and Yan 2017).

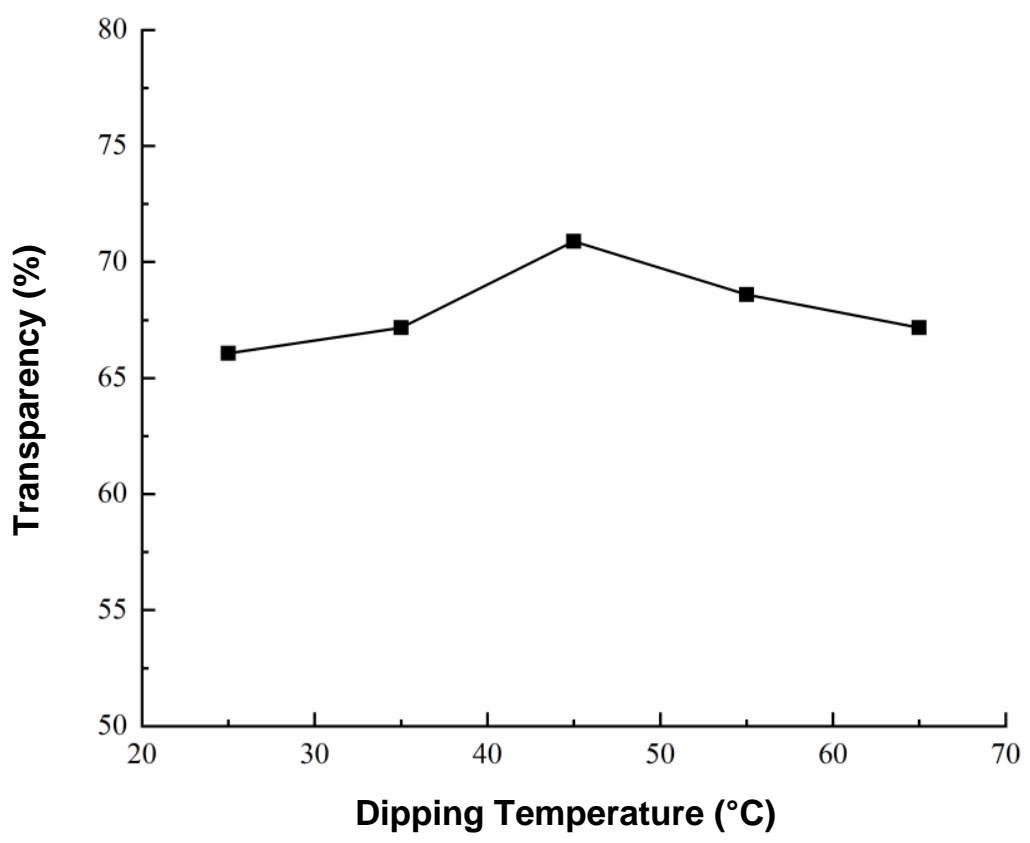

Fig. 4. Effect of dipping temperature on transparency of paper

\section{Effects of dipping temperature on paper strength property}

Figure 5 shows that the relatively low immersion temperature was beneficial to maintaining the strength of the paper. There was an increasing tensile index of the transparent paper at 25 to $35{ }^{\circ} \mathrm{C}$ of dipping temperature, allowing for maintaining the transparent paper at a higher level.

The increase of dipping temperature would deteriorate the transparency performance, due to the quick-drying rates at high temperature (Huang and Petermann 2015). This phenomenon could happen possibly because at high dipping temperature $\left(>35^{\circ} \mathrm{C}\right)$ the fibers of the paper started to be softened and then decreased the paper strength (Kouko and Retulainen 2014). Similar results can also be found in the tear and burst index. 


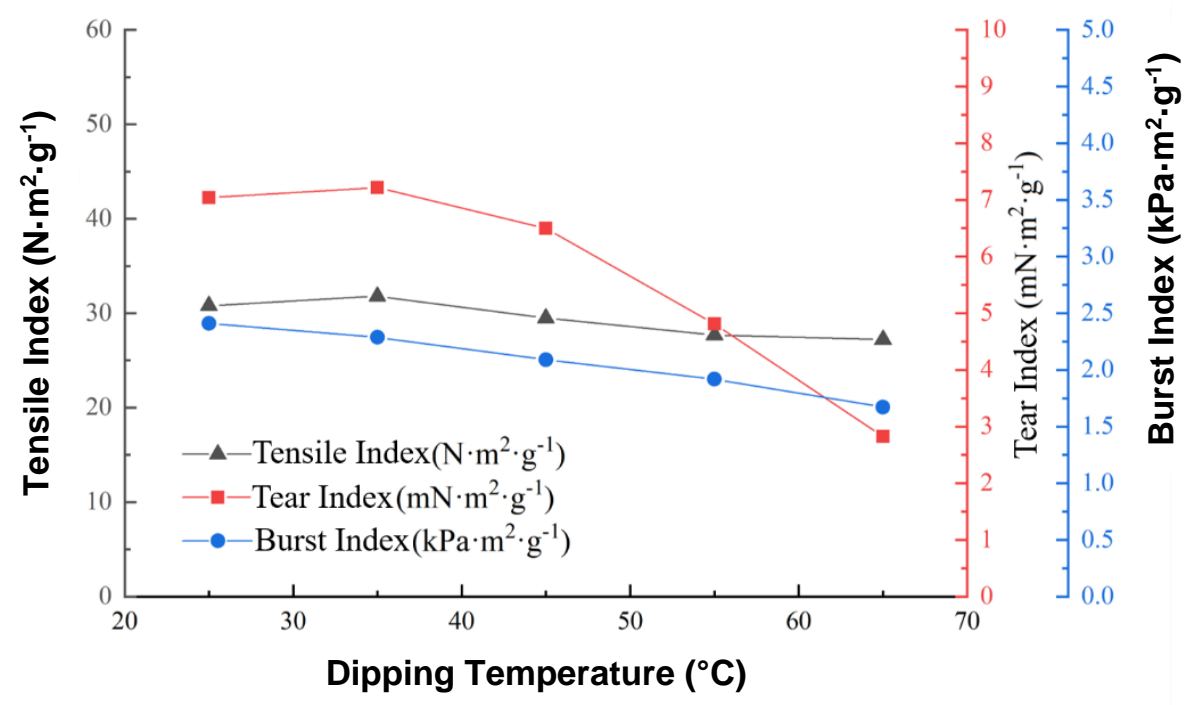

Fig. 5. Effects of dipping temperature on strength of paper

\section{Distribution of Transparentizing Agent in Paper}

The control paper sample and the finished transparent paper were observed in Fig. 6. Figures 6(a) and 6(b) show that the addition of transparentizing agent can improve the surface smoothness of paper, and fill into the voids/ pores between fibers in paper sheet, thus improving the transparency of paper sheet.

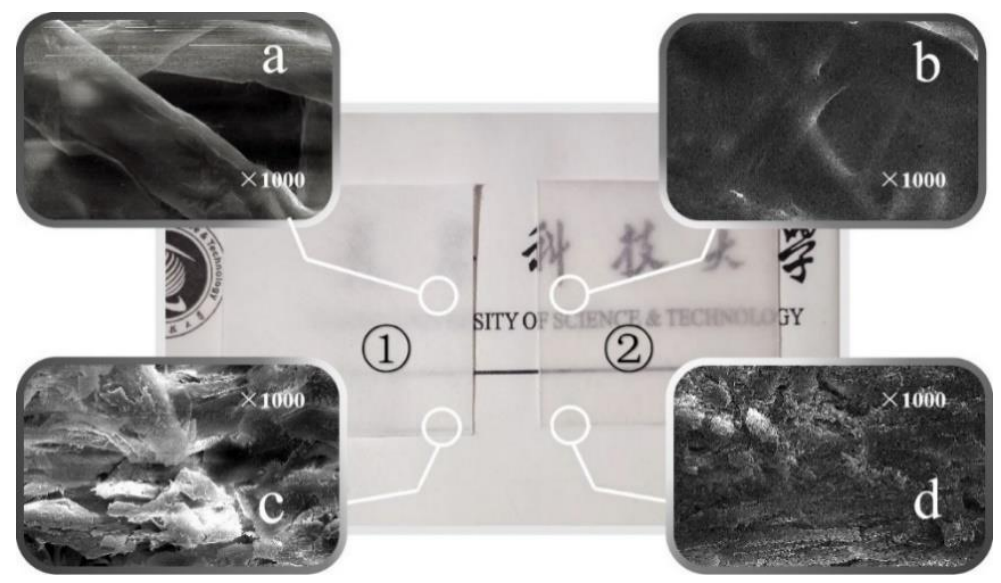

Fig. 6. Comparison of the effect of base paper and transparent paper: (1) base paper, (2) transparent paper, (a) SEM images of the surface of base paper, and (b) SEM images of the surface of transparent paper, (c) SEM images of the cross section of base paper, (d) SEM images of the cross section of base paper

Similar results can also be obtained from the cross section of the paper samples, as shown in Figs. 6(c) and 6(d). From the cross-section of the paper, the transparentizing agent had filled into the pores/voids of paper sheets, there were no apparent boundaries between the fibers, only some small granular substances were loaded on the fiber surface (Bouramtane et al. 2019). 


\section{Stability Analysis of Transparentizing Agent}

There were some key factors affecting the stability of the transparentizing agent emulsions. During the preparation and pumping stages, the transparentizing agent emulsion can be unstable under mechanical shearing treatment. In this case, the transparentizing agent emulsion was processed in a high-speed centrifuge (XiangYi centrifuge instrument Co., Ltd., Tianjin, China) at $3000 \mathrm{rpm}$ for $15 \mathrm{~min}$. It can be concluded that no delamination or precipitation was found in the transparentizing agent emulsions, indicating that the prepared transparentizing agent emulsion possessed excellent mechanical stability.

The transparentizing agent emulsion may be in a high-temperature environment during the storage process, transportation, and process. Therefore, the transparentizing agent was placed in a sealed glass vial and placed in a preheated oven at $60{ }^{\circ} \mathrm{C}$ for 5 days, to ensure that the emulsion will not break in that described above. There was no precipitation, delamination, or demulsification, indicating that the transparentizing agent emulsion can be stored for a long time in a low-temperature environment.

Another method to keep transparentizing agent is to dilute the transparentizing agent emulsion into different concentrations, the lowest to reach a concentration of $0.5 \%$, and place them in test tubes with a liquid column height of $20 \mathrm{~cm}$. The diluted transparentizing agent emulsion was stored at room temperature for more than 30 days without any delamination, precipitation, or demulsification. The test results showed that transparentizing agent emulsion had better dilution stability and storage stability.

\section{Analysis of Biodegradability of Transparency Paper Sheet}

The degradation performance of transparency paper in the soil was investigated, as shown in Fig. 7. This test simulated the laboratory's actual degradation process and analyzed the biodegradability of transparent mulch paper using the soil burial method. The results showed that the paper film's weight loss and strength loss co-occur during the degradation process. After four weeks of burial, the weight loss reached $30 \pm 6.30 \%$, and the strength loss exceeded $50 \pm 3.12 \%$. After being buried for eight weeks, the weight loss reached $52.11 \pm 7.81 \%$ and the strength loss reach almost $100 \%$.

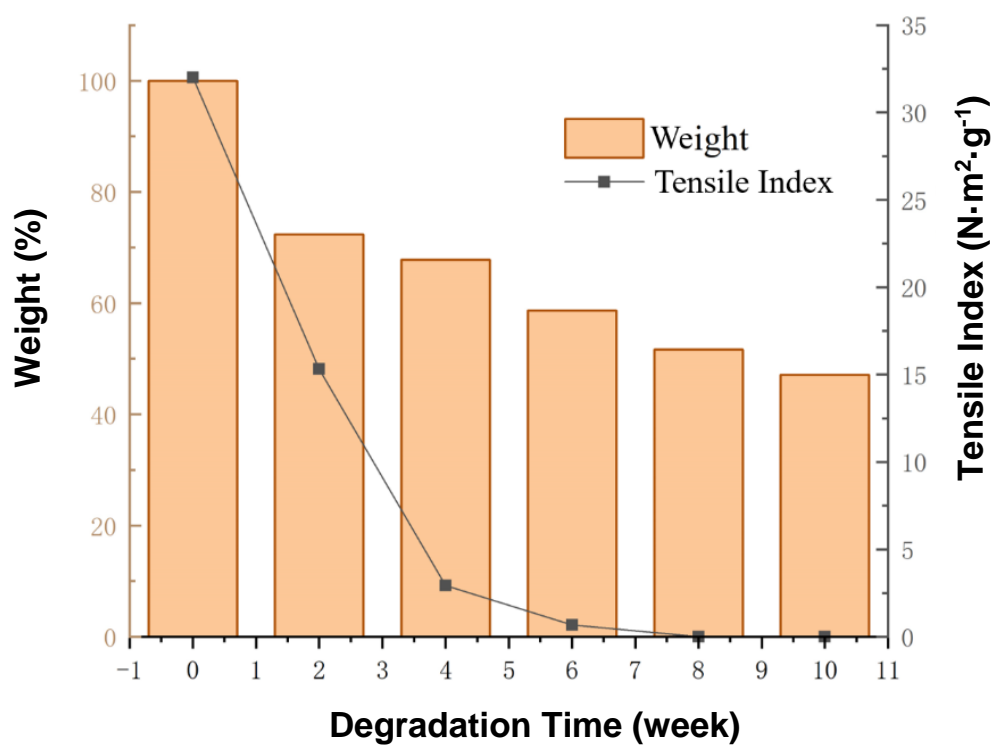

Fig. 7. Effects of degradation time on weight and tensile strength of paper 
The actual degradation process of mulch paper is quite complicated (Ming and Chen 2020). In addition to photodegradation, thermal oxidation, weathering, and hydrolysis, soil also contains many microorganisms, including bacteria, molds, yeasts, and actinomycetes (Alvarez et al. 2006). Some types of bacteria and molds could also produce sufficient amounts of cellulase (Yaacob et al. 2015). The cellulose fibers within mulch paper would be degraded into various products under the above conditions. Those complex degradation products are interrelated to form the entire biodegradation process of transparent paper (Yang et al. 2015), which indicating an excellent biodegradation ability of the prepared mulch paper.

\section{CONCLUSIONS}

In this study, transparentizing agents for transparent paper products have been successfully prepared from natural fiber base paper via a feasible dipping method. The optimum conditions to prepare the transparency paper are: $30 \%$ of transparentizing agent concentration, dipping temperature of 30 to $45^{\circ} \mathrm{C}$, immersion time of $5 \mathrm{~s}$. The prepared transparentizing agent can quickly penetrate the paper structure and the transparency of paper sample can reach as high as $76 \pm 0.97 \%$, which was $37 \pm 1.4 \%$ higher than that of the control. The relative strength properties of the paper were reduced after the application of the transparency agent. The paper transparentizing agent prepared in this paper had a relatively low viscosity, which was beneficial to the dipping process treated via surface sizing device of a paper machine. The transparentizing agent had a better absorption and retention rate in the base paper, thus improving the filling effect in the pores of the base paper, and the paper transparency as well. Biodegradability tests confirmed that the prepared transparent paper possessed an excellent transparent stability and biodegradability. The proposed method for the preparation of transparentizing agent and transparent paper will play a great potential in the replacement of traditional plastic based products, aiming to eliminate the white pollution.

\section{REFERENCES CITED}

Abbasi, F., Mirzadeh, H., and Katbab, A. (2001). "Modification of polysiloxane polymers for biomedical applications: A review," Polymer International 50(12). 1279-1287.

DOI: $10.1002 /$ pi.783

Al-Kayssi, A. W., and Al-Karaghouli, A. (1997). "A new approach for soil solarization by using paraffin-wax emulsion as a mulching material." Renewable Energy 26(4), 637648. DOI: 10.1016/S0960-1481(01)00120-3

Alvarez, V., Ruseckaite, R., and Vazquez, A. (2006). "Degradation of sisal fibre/mater biy biocomposites buried in soil," Polymer Degradation and Stability 91(12), 3156-

3162. DOI: 10.1016/j.polymdegradstab.2006.07.011

Bouramtane, S., Bretin, L., Pinon, A., Leger, D., Liagre, B., Richard, L., Brégier, F., Sol, V., and Chaleix, V. (2019). "Porphyrin-xylan-coated silica nanoparticles for anticancer photodynamic therapy," Carbohydrate Polymers 11(10), 1474.

Cappelletto, E., Callone, E., Campostrini, R., Girardi, F., Maggini, S., Volpe, C., Siboni, S., and Di Maggio, R. (2012). "Hydrophobic siloxane paper coatings: The effect of increasing methyl substitution," Journal of Sol-Gel Science and Technology 62(3), 
441-452. DOI: 10.1007/s10971-012-2747-1

Cender, T. A., Simacek, P., and Advani, S. G. (2013). "Resin film impregnation in fabric prepregs with dual length scale permeability," Composites Part A: Applied and Manufacturing 53, 118-128. DOI: 10.1016/j.compositesa.2013.05.013

Chen, C., Li, D., Deng, Q., and Zheng, B. (2012). "Optically transparent biocomposites: Polymethylmethacrylate reinforced with high-performance chitin nanofibers," BioResources 7(4), 5960-5971. DOI: 10.15376/biores.7.4.5960-5971

Chen, J., Han, X., Fang, Z., Cheng, F., Zhao, B., Lu, P., Li, J., Dai, J., Lacey, S., and Elspas, R. (2015). "Rapid dissolving-debonding strategy for optically transparent paper production," Scientific Reports 5, 17703. DOI: 10.1038/srep17703

Chen, Y., Wan, J., Zhang, X., Ma, Y., and Wang, Y. (2012). "Effect of beating on recycled properties of unbleached eucalyptus cellulose fiber," Carbohydrate Polymers 87(1), 730-736. DOI: 10.1016/j.carbpol.2011.08.051

Cirujeda, A., Aibar, J., Anzalone, A., Martin-Closas, L., Meco, R., Moreno, M. M., Pardo, A., Pelacho, A. M., Rojo, F., Royo-Esnal, A., Suso, M. L., and Zaragoza, C. (2012). "Biodegradable mulch instead of polyethylene for weed control of processing tomato production," Agronomy for Sustainable Development 32, 889-897. DOI: 10.1007/s13593-012-0084-y

Cirujeda, A., Aibar, J., Moreno, M. M., and Zaragoza, C. (2013). "Effective mechanical weed control in processing tomato: seven years of results," Renewable Agriculture and Food Systems, 30(3), 1-10. DOI: 10.1017/S1742170513000434

Fang, Z., Zhu, H., Preston, C., Han, X., Li, Y., Lee, S., Chai, X., Chen, G., Hu, L. (2013). "Highly transparent and writable wood all-cellulose hybrid nanostructured paper," Journal of Materials Chemistry C 1(39), 6191-6197. DOI: 10.1039/C3TC31331J

Guan, F.-X., Song, Z.-P., Xin, F.-R., Wang, H.-L., Yu, D.-H., Li, G.-D., and Liu, W.-X. (2020). "Preparation of hydrophobic transparent paper via using polydimethylsiloxane as transparent agent," J. Biores. Bioprod. 5, 37-43. DOI: 10.1016/j.jobab.2020.03.004

Hämäläinen, J., Eskola, R., Erkkilä, A.-L., and Leppänen, T. (2011). "Rheology in papermaking - from fiber suspension flows to mechanics of solid paper," Korea Australia Rheology Journal 23(4), 211-217. DOI: 10.1007/s13367-011-0026-2

Hassan, E. A., Hassan, M. L., Abou-zeid, R. E., and El-Wakil, N. A. (2016). "Novel nanofibrillated cellulose/chitosan nanoparticles nanocomposites films and their use for paper coating," Industrial Crops and Products 93, 219-226. DOI: 10.1016/j.indcrop.2015.12.006

Huang, L., and Petermann, M. (2015). "An experimental study on rheological behaviors of paraffin/water phase change emulsion," International Journal of Heat and Mass Transfer 83, 479-486. DOI: 10.1016 / j.ijheatmasstransfer.2014.12.037

Hui, L., Zhao, C., Han, W., Jiang, Y., and Wang, S. (2018). "Preparation and research of environment-friendly degradable paper mulching film of cotton stalk fiber," IOP Conference Series: Earth and Environmental Science 199(4). DOI: 10.1088/17551315/199/4/042052

Ibrahim, M., Khan, A., Anjum, Ali, W., and Akbar, H. (2020). "Mulching techniques: An approach for offsetting soil moisture deficit and enhancing manure mineralization during maize cultivation," Soil and Tillage Research 200, 104631. DOI: 10.1016/j.still.2020.104631

Jang, S.-H., Marchal, V., Panigrahi, K. C. S., Wenkel, S., Soppe, W., Deng, X-W., Valderde, F., and Coupland, G. (2008). "Arabidopsis COP1 shapes the temporal 
pattern of $\mathrm{CO}$ accumulation conferring a photoperiodic flowering response," The EMBO Journal 27, 1277-1288. DOI: 10.1038/emboj.2008.68

Koroleva, M. Y., Gorbachevski, O. S., and Yurtov, E. (2017). "Paraffin wax emulsions stabilized with polymers, surfactants, and nanoparticles." Theoretical Foundations of Chemical Engineering 51(1), 125-132. DOI: 10.1134/S0040579516060087

Kouko, J., and Retulainen, E. (2014). "Straining and relaxation properties of wet paper during heating," Mechanics of Time-Dependent Materials, 18(4), 697-719. DOI: 10.1007/s11043-014-9246-4

Li, Z., Liu, W., Guan, F., Li, G., Song, Z., Yu, D., Wang, H., and Liu, H. (2019). "Using cellulose fibers to fabricate transparent paper by microfibrillation," Carbohydrate Polymers 214, 26-33. DOI: 10.1016/j.carbpol.2019.03.019

Mackey, L. N., Ferershtehkhou, S., and Scheibel, J. J. (1994). "Tissue paper treated with polyhydroxy fatty acid amide softener systems that are biodegradable," International Publication No. WO1995016823A1.

Marí, A., Pardo, G., Cirujeda, A., and Martínez, Y. (2019). "Economic evaluation of biodegradable plastic films and paper mulches used in open-air grown pepper (Capsicum annum L.) crop," Agronomy 9(1), 36. DOI: 10.3390/agronomy9010036

Ming, X., and Chen, Q. (2020). "Experiment on cultivation performance of plant fiberbased degradable film in paddy field." Applied Sciences 10(2), 495. DOI: 10.3390/app10020495

Minoru and Yamauchi, M. (2001). "Crop establishment and grain yield of direct sowing culture of rice with recycled-paper mulch (Agronomy)," Japanese Journal of Crop Science 70(2), 164-172. DOI: 10.1626/jcs.70.164

Obasi, H. C., Igwe, I. O., and Madufor, I. C. (2013). "Effect of soil burial on tensile properties of polypropylene/plasticized cassava starch blends," Advances in Materials Science and Engineering 2013(326538), 5. DOI: 10.1155/2013/326538

Pelton, R. H., Yang, D., and Gustafsson, E. (2019). "Polymers that strengthen never-dried joints between wet cellulose surfaces - A review," BioResources 14(1), 2389-2419. DOI: 10.15376/biores.14.1.Pelton

Shen, L., Wang, P., Zhang, L. (2011). "Effects of degradable film on soil temperature, moisture and growth of maize," Nongye Gongcheng Xuebao/transactions of the Chinese Society of Agricultural Engineering 27(6), 25-30. DOI: 10.3969/j.issn.10026819.2011.06.005

Su, Y., Yang, B., Liu, J., Sun, B., Cao, C., Zou, X., Lutes, R., and He, Z. (2018). "Prospects for replacement of some plastics in packaging with lignocellulose materials: A brief review," BioResources 13(2), 4550-4576. DOI: 10.15376/biores. 13.2.4550-4576

Syed, N. F. N., Zakaria, M. H., and Bujang, J. S. (2016). "Fiber characteristics and papermaking of seagrass using hand-beaten and blended pulp," BioResources 11(2). DOI: 10.15376/biores.11.2.3558-5380

Taiki, F., and Masami, K. (2012). "Characterization of paraffin oil emulsions stabilized by hydroxypropyl methylcellulose," Journal of Colloid and Interface Science 367(1), 55-60. DOI: 10.1016/j.jcis.2011.10.034

Tang, X., and Yan, X. (2017). "Dip-coating for fibrous materials: mechanism, methods and applications," Journal of Sol-Gel Science and Technology 81(2), 378-404. DOI: 10.1007/s10971-016-4197-7

Tiwari, I., and Mahanwar, P. A. (2018). "'Polyacrylate/silica hybrid materials: A step towards multifunctional properties," Journal of Dispersion Science and Technology 
40(7), 1-33. DOI: 10.1080/01932691.2018.1489276

Vitz, J., Yevlampieva, N. P., Rjumtsev, E., and Schubert, U. S. (2010). “Cellulose molecular properties in 1-alkyl-3-methylimidazolium-based ionic liquid mixtures with pyridine," Carbohydrate Polymers 82(4), 1046-1053. DOI:

10.1016/j.carbpol.2010.06.016

Yaacob, N. D., Ismail, H., and Sung Ting, S. (2015). "Soil burial of polylactic acid/paddy straw powder biocomposite," BioResources 11(1), 1255-1269. DOI: 10.15376/biores.11.1.1255-1269

Yang, N., Sun, Z., Feng, L., Zheng, M., Chi, D., Meng, W., Hou, Z., Bai, W., and Li, K. (2015). "Plastic film mulching for water-efficient agricultural applications and degradable films materials development research," Advanced Manufacturing Processes 30(2), 143-154. DOI: 10.1080/10426914.2014.930958

Zhang, W., Lu, P., Qian, L., and Xiao, H. (2014). "Fabrication of superhydrophobic paper surface via wax mixture coating," Chemical Engineering Journal 250, 431-436. DOI: 10.1016/j.cej.2014.04.050

Zhou, S., Li, Y.-L., Huang, L.-L., Chen, L.-H., and Miao, Q.-X. (2018a). "Enhanced reactivity of kraft-based viscose-grade dissolving pulp by Hollander beating treatment," BioResources 13(2), 2861-2870. DOI: 10.15376/biores.13.2.2861-2870

Zhou, P., Zhu, P., Chen, G., Liu, Y., Kuang, Y., Liu, Y., and Fang, Z. (2018b). "A study on the transmission haze and mechanical properties of highly transparent paper with different fiber species," Cellulose 25(3), 2051-2061. DOI:10.1007/s10570-018-1663-0

Zhu, H., Fang, Z., Preston, C., Li, Y., and Hu, L. (2014). "Transparent paper:

Fabrications, properties, and device applications," Energy and Environmental Science 7, 269-287. DOI: 10.1039/C3EE43024C

Article submitted: August 31, 2020; Peer review completed: October 4, 2020; Revised version received and accepted: October 31, 2020; Published: November 16, 2020.

DOI: 10.15376/biores.16.1.277-290 